\title{
Orquestar la Evaluación Inclusiva en los Centros Educativos. ¿Por dónde Empezar?
}

\section{Orchesting Inclusive Evaluation in Schools. Where We Could Start?}

\author{
$\mathrm{M}^{\mathrm{a}}$ Amparo Calatayud * \\ Universitat de València, España
}

\begin{abstract}
Es cierto que en los últimos años se ha avanzado bastante en un discurso de la inclusividad potente y fundamentado, pero las prácticas siguen la inercia de una evaluación que parte de que todos los estudiantes son iguales. Se sigue arrastrando una cultura evaluativa asentada en que el aprendizaje está al servicio de la evaluación y la enseñanza termina subordinada a esta última. Una cultura reduccionista y calificadora muy arraigada, resistente al cambio y que cuesta mucho de reconstruir y transformar hacia una evaluación para la inclusión. En teoría hemos pasado de una evaluación centrada en la comprobación del saber, la certificación, el control, la calificación, etc. y en la que todos los alumnos son iguales a una consideración de la evaluación como oportunidad para el aprendizaje y en la que cada estudiante es único y diferente. Es por ello que, en este artículo se intente articular las genuinas cartas náuticas hacia la inclusividad real en los centros educativos, incidiendo, posteriormente, en una de esas cartas, la relativa a la evaluación inclusiva para finalizar con la presentación de algunas de las estrategias que pueden hacer realidad esta evaluación en las aulas inclusivas.
\end{abstract}

Descriptores: Evaluación inclusiva; Mejora; Aprendizaje; Diversidad; Retroalimentación.

It is true that in recent years a lot of progress has been made in a discourse of powerful and informed inclusivity, but the practices follow the inertia of an evaluation that starts from the fact that all students are equal. It continues to drag an evaluative culture based on the fact that learning is at the service of evaluation and teaching ends up subordinated to the latter. A deep-rooted reductionist and qualifying culture, resistant to change and that costs a lot to rebuild and transform towards an assessment for inclusion. In theory, we have gone from an evaluation focused on the verification of knowledge, certification, control, qualification, etc. and in which all students are equal to a consideration of the evaluation as an opportunity for learning and in which each student is unique and different. That is why, in this article we try to articulate the genuine nautical charts towards real inclusiveness in the educational centers, subsequently influencing in one of those letters, the one related to the inclusive evaluation to end with the presentation of some of the strategies that can make this assessment a reality in inclusive classrooms.

Keywords: Inclusive evaluation; Improvement; Learning; Diversity; Feedback.

*Contacto: amparo.calatayud@uv.es

ISSN: 2254-3139

www.rinace.net/riejs/

revistas.uam.es/riejs
Recibido: $\quad 1$ de agosto 2019

$1{ }^{\text {a }}$ Evaluación: 10 de octubre 2019

$2^{\text {a }}$ Evaluación: 26 de octubre 2019

Aceptado: $\quad 3$ de noviembre 2019 


\section{Introducción}

Dar respuesta a la diversidad supone adentrarse en muchos cambios tanto curriculares, pedagógicos, de gestión, de organización, etc. para atender a las diferencias y necesidades de todos y cada uno de los estudiantes (Agut, 2010). Se reconoce, por suerte, cada vez más la necesidad y la exigencia de una educación inclusiva que atienda a cada alumno en función de sus necesidades específicas o características personales. Ahora bien, los profesionales de la educación debemos de tener muy claro que el éxito de la educación inclusiva radica primordialmente en la evaluación. Un sistema de evaluación que no valore y respete las diferencias individuales sólo conduce a profundizar en la brecha de la desigualdad. La evaluación no puede tener la finalidad exclusiva de clasificar o comparar a los estudiantes sino tratar de identificar la ayuda y los recursos que necesitan para facilitar el proceso de enseñanza y aprendizaje. Además, como comentan Monereo (2009) la evaluación tiene que tener eminentemente una función educadora que debe lograr que aquellos estudiantes que no "aprenden solos" lo hagan y desarrollen al máximo sus potencialidades.

Evaluar para la inclusión no es una tarea fácil porque significa atender a la diversidad del alumnado, en cuanto a: 1) diferencias generales (estilos cognitivos, ritmos de aprendizaje, nivel madurativo, intereses, motivaciones, inteligencias múltiples, etc.), 2) en cuanto a diferencias de capacidad (si son estudiantes de altas capacidades, de dificultades de aprendizaje, etc.) y 3) en cuanto a diferencias por razones sociales y de salud. Y practicar una evaluación inclusiva supone respetar y valorar las diferencias individuales y grupales. Tal y como señalan Anijovich y Cappelletti (2017), si las condiciones de los estudiantes siempre son diferentes, si los ritmos y las formas de aprendizaje son diversos, si los puntos de partida nunca son homogéneos, lo que se aprende y lo que se evalúa no puede estar estandarizado, sino que debe ser diferenciado de acuerdo con las condiciones y los procesos individuales de los estudiantes.

En este artículo se pretenden articular las cartas náuticas hacia la inclusividad real en los centros educativos, incidiendo en una de esas cartas, la relativa a la evaluación inclusiva para finalizar con la presentación de algunas de las estrategias que pueden hacer realidad esta evaluación en las aulas inclusivas.

\section{Las cartas náuticas hacia la inclusividad}

Las cartas náuticas son cartas de navegación marítima que muestran todo tipo de argumentos (tipo de fondo, roca, arena, zonas de peligro, etc.) para ayudar en la navegación o para llevar a cabo una navegación segura. A continuación, se plantean las 10 cartas más determinantes hacia la inclusividad, Después nos centraremos en aquella que se considera que marca el rumbo de la educación inclusiva: la evaluación.

Algunas de esas cartas náuticas deberían de empezar por:

- Cuestionar nuestras creencias y prácticas educativas para reflexionar sobre si lo que estamos haciendo está posibilitando la inclusión. Los docentes resignifican, interpretan y construyen su saber profesional, a través de procesos de reflexión en la acción y reflexión sobre la acción. La reflexión sobre la práctica se convierte en el motor para ser consciente de lo que tenemos que continuar haciendo porque lo hacemos bien y de lo que tenemos que ir cambiando para convertirnos en 
mejores profesionales de la educación facilitadores de la inclusión. Por tanto, como señalan Anijovich y Cappelletti (2017), "es necesario revisar y problematizar en primera instancia, las creencias que subyacen a los enfoques tradicionales en los que se basan las prácticas habituales de evaluación" (p. 41).

- Conocer bien al estudiante, en cuanto cómo aprende, qué le motiva, qué intereses y necesidades tiene. Lo que el alumno aprenda tiene que tener sentido en su entorno, le tiene que ser útil y le tiene que permitir transferirlo a otras situaciones o experiencias. Como afirman Anijovich y Cappelletti (2017), se trata de una forma de aprender que busca que "ese aprendizaje tenga valor no sólo en el ámbito escolar y que sea genuino” (p. 111).

- Descubrir y potenciar las peculiaridades y potencialidades que presenta cada alumno. Cada estudiante necesita ser el campeón de algo. Hay que ayudarle a descubrir sus virtudes y a partir de ahí potenciar todo lo demás. Como señala Pérez Gómez (2019), "nuestro compromiso profesional es ayudar a cada aprendiz a construir su propio y singular proyecto vital hasta el máximo de sus posibilidades" (p. 11).

- Adoptar propuestas metodológicas inclusivas basadas en redes conceptuales, enseñanza constructivista, diálogo socrático, significativo, de descubrimiento, conectivo, cooperativo, por proyectos, de resolución de problemas e investigación. Ello supone, como señala Onrubia (2004), adoptar los criterios psicopedagógicos idóneos para atender a la diversidad de alumnado. Y esos criterios pasan por adecuar las metodologías a los ritmos de aprendizaje de los estudiantes.

- Mirar y practicar la evaluación desde los ojos de la inclusividad. La evaluación ha de entenderse como recurso para mejorar las oportunidades de aprendizaje.

- Considerar el carácter flexible del curriculum para adaptarlo a las características y necesidades de los estudiantes. El docente ha de tener muy claro cuáles son las competencias básicas que se espera que los estudiantes adquieran y los distintos niveles de dominio para cada uno de ellos. Además, es de destacar que la evaluación promueve cambios, como indica Monereo (2009), en los modos en que los alumnos enfocan su aprendizaje, pero también en la manera en que los profesores orientan su enseñanza.

- No olvidar que cada estudiante es único e irrepetible. Descubrir el valor de cada estudiante nos conduce a respetar sus ritmos de aprendizaje, su maduración, sus estilos en la manera de aprender, los conocimientos previos de los que parte. Si no se identifican todos estos aspectos no podemos valorar el progreso del estudiante ni tampoco prever la ayuda que necesita para su aprendizaje.

- Trabajar de forma conjunta entre los docentes, potenciando prácticas educativas interactivas, estrategias de trabajo cooperativo, por proyectos, programación multinivel, etc. que faciliten la planificación, el desarrollo y la evaluación de situaciones ricas de inclusividad.

- Cambios importantes en la organización y en la gestión de los centros. La institución educativa ha de tener la autonomía suficiente para organizarse de la mejor manera posible para fomentar la enseñanza y la evaluación inclusiva. 
- La inspección educativa y los equipos directivos han de asumir un liderazgo para la inclusión. Además, han de facilitar, asesorar, ayudar, flexibilizar estrategias y recursos que potencien los procesos inclusivos. Por ejemplo, aportar protocolos y documentos que faciliten la acción del profesorado.

\section{3. ¿De dónde venimos? De una evaluación que parte de que todos los estudiantes son iguales}

Durante mucho tiempo e incluso me atrevería a decir que, en muchos de nuestros Institutos de Educación Secundaria de España, aún persiste la evaluación dirigida casi exclusivamente a medir resultados finales de aprendizaje. La función social de la evaluación ha sido mucho más determinante que la función educativa, de análisis de los procesos, de diagnóstico, de comprensión, diálogo, retroalimentación y de participación activa del alumnado en el proceso evaluativo.

La evaluación, en esta cultura, es considerada como un instrumento de control, de comprobación de los aprendizajes, especialmente conceptuales, que se identifican por medio de pruebas escritas. En este sentido, los autores Mellado y Chaucono (2015) determinan este enfoque evaluativo como la medición de los resultados de aprendizaje al final de un periodo de ejecución de la enseñanza para verificar el dominio de contenidos y calificar con el propósito de acreditación. Por tanto, el único agente evaluador es el profesorado. Además, se le otorga una escasa retroalimentación y se concibe el error con una connotación negativa. Por tanto, el aprendizaje está al servicio de la evaluación y la enseñanza termina subordinada a esta última.

Esta concepción de la evaluación vinculada directamente con la valoración de los resultados del aprendizaje de los estudiantes y mediatizada por una racionalidad propia de la perspectiva tecnoburocrática del currículum condiciona practicas evaluativas asentadas en los siguientes rasgos:

- Prevalece la observación de los resultados más que la valoración de los procesos.

- Sólo se evalúan contenidos conceptuales.

- Evaluación comprobativa (si los objetivos previstos se han alcanzado).

- Evaluación continua con carácter sumativo.

- La evaluación como instrumento coercitivo, punitivo...

- Evaluación finalista.

- Uso casi exclusivo del examen.

- El culpable del fracaso escolar es el alumno.

- Objeto de evaluación es exclusivamente el discente.

- No se negocian los criterios de evaluación con los alumnos.

- La recuperación es la repetición del examen.

- Evaluar es sinónimo de calificar, medir, ...

- Se menosprecia el uso de la autoevaluación como elemento de reflexión, desarrollo y madurez. 
- La referencia al control/examen para tomar las decisiones de promoción.

- En las sesiones de evaluación se "cantan" las notas.

- No se asume la importancia del error como fuente de aprendizaje.

- Evaluación para detectar lo negativo.

- Evaluación puntual.

- Evaluación con carácter explícito (el ritual que supone la realización de la evaluación)

- -Se les otorga más peso a las evaluaciones de tipo formal.

- Se ignora el valor de la evaluación del proceso de enseñanza y aprendizaje como instrumento de mejora de la Institución Escolar.

\section{3. ¿Hacia dónde vamos?: Una evaluación para la inclusión}

Una evaluación favorecedora de los procesos de inclusión apuesta por primar la función de diagnóstico, de regulación y adaptación de la planificación del proceso de enseñanza y aprendizaje a las necesidades y dificultades de los estudiantes. Además, incide en que es fundamental emplear diversas técnicas de evaluación, igualmente activas y participativas, no sólo para que el docente las conozca, sino también para que los estudiantes, protagonistas de sus propios aprendizajes puedan tener conocimiento de aquello que saben y hasta dónde pueden llegar, generando en ellos una mentalidad de crecimiento a través de la evaluación. Por tanto, se trata de una evaluación que está al servicio de los aprendizajes y sirve tanto al estudiante como al profesor. Además, facilita la autorregulación del aprendizaje a través de la retroalimentación oportuna y constante del docente.

Concepción evaluativa basada en las directrices de la perspectiva práctica-critica del curriculum y rotundamente vinculada a los procesos, así como en plantear el carácter ideológico de la evaluación, bajo determinantes constructivistas y de pensamiento crítico. Evaluación que debe ser antes que nada un medio para formar al estudiante en su progreso individual y desarrollo del proceso de enseñanza y aprendizaje.

Por tanto, se trata de una evaluación que está integrada dentro del proceso de enseñanza y aprendizaje y no separada o desconectada de él. Planteamiento que se opone al que concibe la evaluación como una práctica que se efectúa al final de un periodo más o menos prolongado de enseñanza, o al término de la realización de alguna unidad temática mediante un acto formal explícito de comprobación, como es el poner una prueba o realizar un examen. Se intenta que la evaluación no sólo sea un acto que conduzca a poner una nota, sino que, sobre todo, ésta se entienda como un proceso de comprensión, de diálogo, de motivación hacia el aprendizaje tanto del profesor como de los alumnos. Una evaluación, a fin de cuentas, como dice Fernández Pérez (1986) que eduque, que nos enseñe "qué es debido a qué", una evaluación formativa y no únicamente sumativa.

En este sentido, una evaluación para que sea realmente inclusiva, emancipadora e iluminativa tiene que ser concebida como: 
- Un proceso crucial para no convertir las diferencias naturales de los estudiantes en desigualdades. Por tanto, ha de partir, como señalan Giné y Piqué (2007), de la certidumbre de que todo estudiante es capaz de aprender, que tiene potencialidades (hay que focalizar la atención en ellas en lugar de hacerlo en las dificultades) y en la premisa de que realmente merece la pena el que le prestemos toda la atención y la ayuda que necesita para que consiga los aprendizajes.

- Una estrategia que ofrezca retroalimentación a los estudiantes para ayudarles en el camino hacia alcanzar su autonomía personal. Para Anijovich y Cappelletti (2017), un estudiante es autónomo cuando comprende el sentido de aquello que tiene que aprender, toma decisiones acerca de cómo va a llevar adelante ese aprendizaje y reflexiona sobre su proceso, su recorrido. La retroalimentación tiene que ir acompañada de orientaciones que le faciliten al estudiante reconocer sus debilidades y fortalezas, reconocer los obstáculos acontecidos, así como prever los modos para abordarlos.

- Un recurso de motivación. Impregnar la evaluación de motivación supone reconocer los grandes o pequeños avances de cada uno de los estudiantes en el proceso de aprendizaje. Se trata de apostar por la evaluación como motivación para aprender.

Indudablemente, desarrollar una evaluación para la inclusividad requiere de unos requisitos, algunos de ellos son:

- Una evaluación que esté insertada en el proceso de enseñanza y aprendizaje. La evaluación y la enseñanza se han de entender y practicar como inseparables. Son dos caras de una misma moneda.

- Una evaluación que incida en el proceso, formativa, procesual, continua y formadora. Una evaluación para el aprendizaje, orientada a pensar en la evaluación como un proceso que tiende a ser continuo y que destaca, sobre todo, el efecto retroalimentador para tratar de que el estudiante avance a partir de sus posibilidades e individualidades.

- Una evaluación que se haga con la finalidad básica de obtener información del alumnado, del proceso y del contexto de aprendizaje, con el fin de mejorarlos. Que sea, en definitiva, útil para tomar decisiones encaminadas hacia la mejora del proceso. En este sentido Onrubia (2004) y Orozco y Moriña (2019) matizan que una de las funciones más importantes de la evaluación, desde el punto de vista de la diversidad del alumnado, es permitir que el docente conozca los distintos recorridos y momentos de aprendizaje por los cuales pueden estar pasando los distintos estudiantes, con el objetivo de poder ofrecer una ayuda tan ajustada como sea posible en cada caso.

- Una evaluación holística. Evaluación de conceptos, procedimientos, actitudes competencias, emociones, motivación, interés, etc.

- Una evaluación que reconozca los esfuerzos realizados por los estudiantes. Es crucial el reconocer los avances individuales respecto al punto de partida especifico. Pero para ello, hay que saber identificar muy bien el punto de partida de cada estudiante. 
- Una evaluación que sea respetuosa con la persona y en la que se pone en el centro al estudiante.

- Una evaluación que utilice diversidad de instrumentos para evaluar el aprendizaje del alumnado. Como señala, Sanmartí (2012),

Dado que los estudiantes son diferentes, es importante diversificar los instrumentos de evaluación. Cada uno estimula unas determinadas habilidades y se adapta más o menos a los estilos de aprender y de enseñar, por lo que variándolos hay más posibilidades de potenciar las cualidades de todos y favorecer el desarrollo de las que no se tienen. (p. 107)

- Una evaluación dentro de lo posible que sea ética, basada en compromisos explícitos que aseguren la cooperación y la aceptación de las personas implicadas. En este sentido, los criterios de evaluación serán públicos, explicitados y negociados con los estudiantes.

- Una evaluación que sea coherente con los contenidos, la enseñanza y las actividades realizadas en clase ("Dime cómo enseñas y te diré cómo evalúas). Como señala Monereo (2009), "la evaluación tiene un peso privilegiado y para la mayoría de los alumnos, constituye la principal razón de las prácticas de estudio y aprendizaje" (p. 15).

- Una evaluación que presente distintos niveles de dificultad. Por tanto, tiene que ser diversificada y flexible. Se han de ofrecer múltiples oportunidades para que los estudiantes demuestren sus avances y lo que realmente han comprendido y aprendido.

- Una evaluación contextualizada, centrada en si el estudiante es capaz de relacionar lo aprendido con otros conocimientos, si es capaz de trasladarlo a situaciones distintas a aquellas que generaron el aprendizaje original. Para ello, una evaluación favorecedora de procesos inclusivo ha de proponer situaciones del mundo real o cercanas a los estudiantes, problemas que sean significativos, complejos para que utilicen sus conocimientos previos, pongan en juego estrategias y demuestren la comprensión de sus saberes.

- Una evaluación que favorezca la reflexión, la innovación, la creatividad, así como el pensamiento crítico y lateral.

- Una evaluación que fomente el trabajo cooperativo y las relaciones socioafectivas.

- Una evaluación como señalan Giné y Piqué (2007) que de "importancia al hecho de aprender". Ello significa que hay que evaluar para corroborar que efectivamente el alumnado está aprendiendo para ver cuál es el progreso que está realizando, para conocer qué conocimientos está adquiriendo y cuáles no ha aprendido. Siendo la evaluación, la clave para establecer un plan de actuación que se adecue a las necesidades de cada estudiante. No hay que olvidar que la evaluación inclusiva ante todo ha de permitir hacer un seguimiento personalizado del alumno y adaptar los contenidos, competencias, estrategias, recursos, etc. a sus concretas necesidades.

- Una evaluación bien diseñada. Lo que significa, el tratar de explicitar a los estudiantes adónde se quiere llegar, qué se pretende que se estudie, el cómo y el 
para qué y qué se requiere para conseguir un proceso de enseñanza y aprendizaje exitoso. Es decir, poner las cartas encima de la mesa.

- Una evaluación que plantea la necesidad de evaluar también lo positivo y no sólo lo negativo para motivar y reconocer que hay aspectos que los estudiantes hacen "bien" pero hay otros que son mejorables.

- Una evaluación que incorpora la autoevaluación y la coevaluación. Se promueve que el estudiante participe activamente y reflexione sobre su propio proceso de aprendizaje.

\section{Estrategias que pueden posibilitar prácticas evaluativas inclusivas en los centros educativos}

Hacer realidad una evaluación inclusiva supone vertebrar unos recursos flexibles y diversificados que incidan en atender a la diversidad del alumnado y, a la vez, ofrecer las mismas oportunidades. Algunos instrumentos para su viabilidad pueden ser los siguientes:

Actividades prácticas que pretendan fomentar en los estudiantes la capacidad de juicio autónomo, de disentir y discrepar, de buscar soluciones personales a los problemas. No se puede caer en la trampa, de la respuesta convergente y correcta. Desde esta visión, se han de proponer a los estudiantes un sistema de trabajo que les permita desplegar su capacidad de comprensión y reflexión sobre las ideas expuestas en clase y las lecturas realizadas partiendo de las características individuales de cada alumno. Actividades que facilitan una "evaluación situada”, en la realidad cercana al estudiante y en la que se establece un vínculo entre escuela y vida.

Pruebas de aprendizaje, con el objeto de conocer si los estudiantes comprenden y transfieren los contenidos fundamentales de cada uno de los temas. Instrumentos que recogen, por un lado, la sistematización de los conocimientos y, por otro, su organización interna. Para ello es necesario el que trabajen a partir de mapas conceptuales, rúbricas, etc. con el fin de comprender las estrategias que pone en marcha cada estudiante.

Trabajos de investigación, se pretende que cumplan la misión de indagación, de investigación, etc. Se valora el rigor en las argumentaciones, la coherencia, la capacidad de reflexión crítica, la utilización de bibliografía adecuada, el uso del lenguaje correcto, la presentación, etc. En este tipo de instrumentos se intenta valorar el grado de integración del estudiante en el grupo, potenciando un aprendizaje cooperativo.

La autoevaluación del estudiante como estrategia formadora, de responsabilización y reflexión del proceso de aprendizaje, si en teoría el protagonista de la educación es el alumno, por lógica, no se le puede negar en la práctica que participe en su proceso de enseñanza y aprendizaje de una manera activa, así como también, en el proceso de evaluación a través, por ejemplo, de la elaboración de instrumentos evaluativos. Concretamente para Boud (1995) se habla de autoevaluación cuando los estudiantes toman la responsabilidad de controlar y hacer juicios sobre aspectos de su propio aprendizaje. Este proceso sugiere, animar a los estudiantes a buscar por sí mismos y en otras fuentes la determinación de criterios que deberán de utilizar para juzgar su propio trabajo, en lugar de dejarse llevar y ser dependientes de los criterios elaborados por sus profesores (Zimmerman y Schunk, 2011). En este sentido, la autoevaluación es una forma de implicar al estudiante en su propio proceso formativo, ya que fomenta la reflexión y la autocrítica sobre el mismo proceso. 
Ahora bien, como señalan Anijovich y Cappelletti (2017), la autoevaluación requiere el desarrollo de determinadas habilidades para que un estudiante pueda juzgar su propio aprendizaje. Especialmente, ha de desarrollar la habilidad para reflexionar sobre lo que ha logrado, lo que es correcto y lo que tiene que mejorar. No hay que olvidar que lo más importante en el proceso de evaluación es que el estudiante aprenda a autoevaluarse.

El portafolio, instrumento para la evaluación inclusiva del estudiante. El portafolio es un método de evaluación del alumnado, alternativo a los métodos tradicionales. Viene a ser como una colección de trabajos, actividades, etc. que el estudiante ha realizado durante un curso. Quizás, lo más importante de esta selección de actividades radique en las siguientes premisas: deben de tratar de evidenciar los esfuerzos realizados por el estudiante, la valoración del trabajo conseguido (¿qué sabia?, ¿qué se yo ahora?, ¿cómo lo he aprendido?, en relación a contenidos tanto del ámbito conceptual, procedimental y actitudinal, así como las competencias aprendidas), cuáles han sido las mejores ideas, los logros conseguidos en los distintos ámbitos de conocimiento y, sobre todo, el portafolio debe de estar compuesto por aquellas actividades que han permitido al estudiante tanto la posibilidad de valorarse más a sí mismos, como de sentirse más seguros de sí mismos. Como indica Díaz Barriga (2006), el portafolio no es una colección al azar o de trabajos sin relación, sino que ha de mostrar el crecimiento gradual y los aprendizajes logrados por los estudiantes en relación con el proceso de enseñanza y aprendizaje realizado.

Indudablemente, esta forma de practicar la evaluación conlleva necesariamente una perspectiva también distinta de pensar y desarrollar el proceso educativo. Implica una metodología basada en el aprendizaje constructivo, en el fomento de la creatividad, la reflexión, la colaboración, la actividad, la participación, etc. que posibilite al estudiante progresar en su madurez y conseguir un equilibrio personal y una integración social.

Como señala Sanmarti (2012), el portafolio es un instrumento que posibilita la autorreflexión y la autogestión del propio trabajo, ya que: 1)“muestra el camino seguido por cada estudiante y hasta dónde ha llegado”; 2) estimula la toma de conciencia y la toma de decisiones; 3) promueve la interrelación entre el pensamiento y la acción y entre la teoría y la práctica; 4) facilita la interacción entre alumnos y entre profesor y alumnos y 5) posibilita que el estudiante visualice pruebas de sus logros.” (p. 109).

Las rúbricas, siguiendo a Anijovich y Cappelletti (2017), se consideran como "asistentes de la evaluación y las define como documentos que articulan las expectativas ante una tarea o un desempeño a través de una lista de criterios y la descripción de sus niveles de calidad" (p. 106) El uso de este instrumento nos va a facilitar el aprendizaje, dado que determina qué se espera y, a su vez, constituye una guía de evaluación tanto para el alumnado como para el profesorado. Concretamente, para los autores Panadero y Romero (2014), las rúbricas resultan ventajosas porque:

- reducen la subjetividad del docente,

- orientan como mapas de rutas, acerca de cómo avanzar en el aprendizaje,

- aportan transparencia al explicitar, a través de los descriptores, los distintos niveles de calidad de los desempeños y las producciones,

- permiten que el estudiante se autoevalúe y promueva la evaluación entre pares,

- muestra al estudiante las áreas en las que tiene que mejorar, y

- estimula la responsabilidad de los estudiantes. 
Diario de aprendizaje, instrumento que desarrolla la metacognición en el alumnado. Cada estudiante reflexiona sobre lo aprendido, qué dificultades ha tenido, qué puede mejorar, etc.

Dianas de evaluación, que nos ayudan a averiguar cómo está aprendiendo el estudiante, especificando de forma clara qué se quiere evaluar y los indicadores que se van a utilizar.

Estrategias de diálogos reflexivos: conversaciones proactivas, progresivas, de andamiaje: es lo que los autores Anijovich y Cappelletti (2017), definen como interacciones dialogadas formativas. Estas interacciones como denominan estos autores son conversaciones entre docentes y estudiantes cuyo propósito es articular "las evidencias de aprendizaje con los objetivos y los criterios de evaluación” (p.93). Además, se orienta la reflexión sobre el aprendizaje realizado, identificando los obstáculos en la adquisición y los modos de cómo superarlos. Sin lugar a dudas, esta estrategia representa el sentido de la evaluación como fuente de comunicación entre docente y estudiante, en el que se explicitan los errores y se posibilitan estrategias de superación.

\section{Discusión y conclusiones}

A lo largo de este artículo se ha evidenciado que se ha pasado, en teoría, de una evaluación centrada en la comprobación del saber, la certificación, el control, la calificación, etc. y en la que todos los alumnos son iguales, a una consideración de la evaluación como oportunidad para el aprendizaje y en la que cada estudiante es único y diferente. Esto supone abordar la evaluación como herramienta para que todos aprendan y como posibilidad de valorar la propia individualidad del estudiante. Se trata de apostar por una evaluación, como señala Casanova (2011), que esté al servicio del ajuste de la ayuda educativa para todos y para cada uno de los estudiantes.

El pilar básico en el que se asienta la evaluación inclusiva está en reconocer que cada uno de los estudiantes que tenemos en el aula es diferente y que, para ello, se necesita de distintas estrategias para favorecer sus aprendizajes y para encarar la enseñanza y la evaluación de forma diferente y adecuada a las características individuales de cada uno de ellos. Uno de los retos educativos más importantes del siglo XXI está en articular una evaluación capaz de dar cuenta de la diversidad y de la heterogeneidad de estudiantes siendo ésta equitativa y justa para todos y todas y respetando cada una de las individualidades. Como argumenta Pérez Gómez (2019, p. 9), "el gran desafío actual consiste en provocar el desarrollo personalizado y hasta el máximo de sus posibilidades de todos y cada uno de los aprendices, celebrando la diversidad, la singularidad de cada uno, respetando la discrepancia y ayudando de modo especial a quien más lo necesitan". La inclusión, como señalan Torrego y Monge (2018), se convierte en una fuente de oportunidades para todos. Para ello, como hemos comentado, la evaluación ha de cumplir unos requisitos, entre los más relevantes destacamos, los siguientes: una evaluación que esté insertada en el proceso de enseñanza y aprendizaje; una evaluación que incida en el proceso, formativa y formadora; una evaluación holística; que utilice diversidad de instrumentos para evaluar el aprendizaje; que presente distintos niveles de dificultad; una evaluación contextualizada; que favorezca la reflexión, la innovación, la creatividad; que fomente el trabajo cooperativo y que plantee la autoevaluación y la coevaluación, así como, la necesidad de evaluar también lo positivo y no sólo lo negativo. 
A pesar de que ha quedado suficientemente evidenciado que el principal dilema de la educación inclusiva reside en hacer realidad la evaluación inclusiva, también es cierto que responder a este reto supone que el profesorado asuma la responsabilidad de evaluar, desevaluar y reevaluar para no convertir las diferencias individuales naturales de los estudiantes en desigualdades. Es en este reto dónde reside el valor genuino de la evaluación para la inclusión que supone abordarla con garantías de equidad y de justicia social en nuestros centros educativos. Con los supuestos y realidades presentadas en este artículo, pensamos que ya se puede intentar vislumbrar el camino hacia la inclusividad, entre otras razones, porque se han explicitado algunos argumentos de: ¿por dónde empezar? Ahora sólo falta insértalos en nuestras prácticas para hacer realidad la evaluación para la inclusión. Por tanto, ¿̇a qué estamos esperando?

\section{Referencias}

Agut, N. (2010). La evaluación en un modelo de escuela inclusiva. Aula de Innovación Educativa, 191, 42-44.

Anijovich, R. y Cappelletti, G. (2017). La evaluación como oportunidad. Buenos Aires: Paidós.

Díaz Barriga, F. (2006). Enseñanza situada: Vinculo entre la escuela y la vida. Ciudad de México: McGraw-Hill.

Boud, D. (1995). Enhancing learning through self-assessment. Londres: Kogan.

Casanova, M. A. (201 1). Evaluación para la inclusión educativa. Revista Iberoamericana de Evaluación Educativa, 4, 78-89.

Fernández Pérez, M. (1986). Evaluación y cambio educativo. El fracaso escolar. Madrid: Morata.

Giné, N. y Piqué, B. (2007). Evaluación para la inclusión. Siete propuestas en forma de tesis. Aula de Innovación Educativa, 163, 7-13.

Mellado, M. E y Chaucono, J. C. (2015). Creencias pedagógicas del profesorado de una escuela rural en el contexto mapuche. Actualidades investigativas en Educación, 15(3), art 4. https://doi.org/10.15517/aie.v15i3.20924

Monereo, C. (2009). PISA como excusa. Repensar la evaluación para cambiar la enseñanza. Barcelona: Graó.

Onrubia, J. (2004). Criterios psicopedagógicos y recursos para atender la diversidad en secundaria. Barcelona: Graó.

Orozco, I. y Moriña, A. (2019). Práctica docente para una pedagogía inclusiva en educación primaria: Escuchando las voces del profesorado. Aula Abierta, 48(3), art 6. https://doi.org/10.17811/rifie.48.3.2019.331-338

Panadero, E. y Romero, M. (2014). To rubric or not to rubric. The effect of self-assessment on selfregulation, performance and self-efficacy. Assessment in Education Principles, Policy and Practice, 21(1), 133-148. https://doi.org/10.1080/0969594X.2013.877872

Pérez Gómez. A. (2019). Ser docente en tiempos de incertidumbre y perplejidad. Revista Márgenes, 1, 3-17. https://doi.org/10.24310/mgnmar.voio.6497

Sanmartí, N. (2012). 10 Ideas: Evaluar para aprender. Barcelona: Graó.

Torrego, J. C. y Monge, C. (2018). Inclusión educativa y aprendizaje cooperativo. Madrid: Síntesis.

Zimmerman, B. y Schunk, D. (2011). Handbook of self-regulation of learning and performance. Nueva York, NY: Routledge. 


\section{Breve CV de la autora}

\section{$M^{a}$ Amparo Calatayud}

Es Premio Extraordinario de Licenciatura y Doctora en Filosofía y Ciencias de la Educación por la Universidad de Valencia. Ha desempeñado varios puestos docentes: funcionaria de carrera del Cuerpo de Maestros en la Escuela Pública. Asesora de Formación del profesorado no universitario adscrito a la Conselleria de Cultura, Educación y Deportes de la Generalidad Valenciana. Técnica en Evaluación en el Instituto Valenciano de Evaluación y Calidad Educativa de la Conselleria de Educación, Cultura y Deportes y Profesora de la Universidad Nacional de Educación a Distancia en el Centro Asociado Alcira-Valencia. En la actualidad es profesora Titular de Didáctica y Organización Escolar en la Facultad de Filosofía y Ciencias de la Educación de la Universitat de València. En el curso 2009-2010, el Rector de la Universitat de València le concedió el Primer Premio a la Excelencia Docente. Ha participado en investigaciones y pronunciado conferencias sobre cuestiones relacionadas con la evaluación educativa, la dirección y liderazgo, la innovación educativa, la organización y gestión de centros escolares y la formación permanente del profesorado. Además de su trayectoria docente e investigadora ha colaborado y continúa colaborando con Organismos y Agencias Nacionales de la Calidad en Educación y Acreditación adscritos al Ministerio de Ciencia, Innovación y Universidades. ORCID ID: https://orcid.org/0000-0003-3250-4580. Email: amparo.calatayud@uv.es 